\title{
On convergence of the $\chi \mathrm{PT}$ HFF expansion for one loop contribution to meson production in NN collisions
}

\author{
E.Gedalin* A.Moalem ${ }^{\dagger}$ and L.Razdolskaya ${ }^{\ddagger}$ \\ Department of Physics, Ben Gurion University, 84105, Beer Sheva, Israel
}

\begin{abstract}
We consider the application of heavy fermion formalism based chiral perturbation theory to meson production in nucleon-nucleon collisions. It is shown that for one loop contributions the heavy fermion formalism expansion corrections for the nucleon propagator produce infinite series of correction terms which are of the same momentum power order. This destroys the one-to-one correspondence between the perturbative and small momentum expansion and thus negates the application of any finite order heavy fermion formalism chiral perturbation theory to the $N N \rightarrow$ $N N \pi$ reactions.
\end{abstract}

Key Words: hadroproduction, chiral perturbation theory, heavy fermion formalism.

\section{INTRODUCTION}

In recent years, intensive theoretical efforts have been devoted to investigating how nuclear and hadron interactions can be understood within Chiral Perturbation Theory $(\chi \mathrm{PT})$, an approach which is generally believed to be an effective theory of Quantum Chromodynamics (QCD) at low energies. Hopefully, such an approach will provide a clue towards understanding nuclear dynamics within the context of QCD, the accepted fundamental theory of strong interactions.

In accordance with the Goldstone theorem, the low energy strong interactions of colorless particles are dominated by colorless pseudoscalar massless boson octet. Since all interactions of the Goldstone bosons vanish at zero energy, the amplitude of processes (between hadrons) can be expanded in powers of external momenta and quark masses. This expansion amounts to a derivative expansion of the effective Lagrangian which is based on the nonlinear realization of chiral symmetry, so that perturbative expansions of effective field theories reproduce the low momentum expansion of the QCD Green functions. A drawback of this fully relativistic approach is that no one-to-one correspondence between loop contributions and small momentum expansion can be established for processes with nucleons. Such a correspondence is believed to be restored in extremely non-relativistic approximation of the heavy fermion formalism (HFF) based $\chi \mathrm{PT}$ [1,2]. In particular, Cohen et al. [4] proposed a modified power counting for meson production in NN collisions, and a scheme as such was used by several groups [3]- 11] to calculate pion production rate in nucleonnucleon collisions. Namely, Park et al. [3], Cohen et al. [4] and Sato et al. [5] have considered the $p p \rightarrow p p \pi^{0}$ process taking into account contributions from lowest order tree graphs. The leading order impulse and rescattering terms are found to have opposite signs, and hence leading to a cross section substantially smaller than experiment [3 5 . Much of the virtue of these calculations resides on how rapid the HFF expansion converges. Detailed $\chi \mathrm{PT}$ calculations which account for all contributions from tree and one loop diagrams to chiral order $\mathrm{D}=2$, show that within the framework of the HFF, one loop contributions are sizably bigger than the lowest-order impulse and rescattering terms [6, 10], indicating that the HFF power series expansion converges rather slowly and therefore may not be suitable to calculate pion production rate in NN collisions [6]. More recently Bernard et al. [7] and Gedalin et al. [8] have shown that the HFF power series expansion of the nucleon propagator is on the border of its convergence circle, and concluded that a finite order HFF can not possibly predict nucleon pole terms correctly and hence can not explain meson production in $\mathrm{NN}$ collisions.

In this regard, it is to be noted that meson-exchange models as well as a fully relativistic $\chi \mathrm{PT}$ predict equal signs for the impulse and rescattering terms, achieving quite impressive descriptions of data near threshold [12, 14, 6, 15, 16, 8]. Particularly, in covariant exchange models [12,6] the amplitudes from the impulse and rescattering terms interfere constructively. It has been argued [15] that this sign difference between predictions of meson exchange models and HFF $\chi \mathrm{PT}$ is a genuine feature. Tamura et al. [16] have shown that the shape of energy spectra for the $d\left(p,(p p)_{s}\right) \pi^{0} n$ and $d\left(p,(p p)_{s}\right) \pi^{-} p$ reactions, can be explained only if the interference between these terms is constructive. Next, Gedalin et al. [8] have shown that in a relativistic $\chi \mathrm{PT}$ the sign and relative importance of various contributions are different from those found using HFF; the rescattering term is found to be as large and having an equal sign as the impulse term. It was also shown that these differences are mostly an outcome of the fact that the HFF Lagrangian

*gedal@bgumail.bgu.ac.il

${ }^{\dagger}$ moalem@bgumail.bgu.ac.il

${ }^{\ddagger}$ ljuba@bgumail.bgu.ac.il 
provides a reasonable nucleon kinetic term only for energies close to zero and therefore, the validity of the HFF Lagrangian becomes limited to rather small nucleon momenta.

It is the purpose of the present note to show that within the framework of HFF $\chi \mathrm{PT}$ and for sufficiently large momentum transfer processes, the one-to-one correspondence between the perturbative expansion and small momentum expansion is badly destroyed at tree level as well as for one loop contributions. Namely, the HFF expansion corrections for the nucleon propagator form an infinite series of terms all of which having the same momentum power. In Sec. II we examine the influence of HFF nucleon propagator corrections to tree and one loop diagrams. In Sec. III we apply power counting scheme for further examination of the validity of the HFF to pion production in NN collisions. We conclude in Sec. IV.

\section{TREE AND LOOP CONTRIBUTIONS}

To be specific we consider pion production via the $N N \rightarrow N N \pi$ reaction. In the two flavor sector of $\mathrm{HFF} \chi \mathrm{PT}$, the important contribution to the meson production amplitude comes from the (irreducible) diagram 1a, where a virtual pion created on one of the incoming nucleons is converted into a final pion on the second nucleon via a $\pi N \rightarrow \pi N$ conversion process. The characteristic four momentum transferred at threshold is $q \approx(-m / 2, \sqrt{M m})$, with $M$ and $m$ being the masses of the nucleon and meson produced. Here to be emphasized that the corresponding $\pi N \rightarrow \pi N$ amplitude is half off mass shell and involves large momentum transfer. This as will be demonstrated, constitutes a major difficulty implementing $\mathrm{HFF} \chi \mathrm{PT}$ to the production process.

As already mentioned above, in the transition from a relativistic to non-relativistic HFF Lagrangian one reduces the nucleon kinetic term. This affects the nucleon propagator strongly and in turn tree level as well as one loop contributions. We demonstrate that by considering $\mathrm{u}$ and $\mathrm{s}$ nucleon pole terms (diagrams $2 \mathrm{a}$ and $2 \mathrm{~b}$ ) and one loop (diagram 2c) contributions to the $\pi N \rightarrow \pi N$ conversion amplitude. All of these involve the nucleon propagator,

$$
S_{N}(p)=i \frac{\not p+M}{p^{2}-M^{2}}
$$

which we may separate into a positive and negative energy parts. To this aim we write the numerator in Eqn. 1 as

$$
\not p+M=M(1+\not)+(\not p-M \psi)=2 M P_{+}+\not\left(P_{+}+P_{-}\right),
$$

where $v^{\mu}$ denotes the four velocity of the nucleon with $v^{2}=1, l^{\mu}=p^{\mu}-M v^{\mu}$ is its residual momentum, and $P_{ \pm}=(1 / 2)(1 \pm \not)$ are operators which project the nucleon Dirac field into large and small components. Following the usual relativistic to non-relativistic reduction procedure(see for example [1]), we define $p_{\perp}^{\mu}=p^{\mu}-(p v) v^{\mu}$ to be the transversal component of the nucleon momentum and write the nucleon propagator in the form,

$$
S_{N}(l)=i\left[2 M P_{+}+\not\left(P_{+}+P_{-}\right)\right] \frac{1}{2\left(M+T\left(p_{\perp}\right)\right)}\left[\frac{1}{v l-T\left(p_{\perp}\right)}-\frac{1}{2 M+v l+T\left(p_{\perp}\right)}\right],
$$

with $T\left(p_{\perp}\right)=\sqrt{M^{2}+p_{\perp}^{2}}-M$. ( The expression, Eqn. 3, is just a solution of the HFF equations for the nucleon propagator quoted by Park, Min and Rho [1). By making use of this expression the contributions from $\mathrm{s}$ and $\mathrm{u}$ channels (e.g., graphs 2a and 2b) separate into negative and positive energy parts. The former part behave like a regular contact term. For example, the non-relativistic limit of the negative energy part of the nucleon propagator at low kinetic energy reduces to $S_{N} \approx-i P_{+} / 2 M$, and the respective negative energy contributions from graphs $2 \mathrm{a}$ and $2 \mathrm{~b}$ sum up to be,

$$
T_{Z} \approx \frac{g_{A}^{2}}{4 M F^{2}}(v q)(v k) .
$$

This indeed has the form of a contact term. In passing by, we note that by separating the nucleon propagator into negative and positive energy parts the s and u channels (graphs 2a, 2b) split into direct and Z-graph contributions [17]. In the non-relativistic limit the Z-graph contribution appears in exactly the form of Eqn. \&, as a local rescattering term, e.g. the negative energy part of the nucleon pole terms " converts" into a sea-gull contact term.

A serious difficulty in the reduced non-relativistic limit concerns with the non-local positive energy part of nucleon pole terms. It is not always possible to calculate the positive energy part within the frame of HFF. To see this consider the expansion of $S_{N}$, Eqn. 3 as "low momentum" power series. The series expansions of the factors $1 /\left(M+T\left(p_{\perp}\right)\right.$ and $1 /\left(2 M+v l+T\left(p_{\perp}\right)\right)$ converge up to sufficiently high energies. However, the series 


$$
\frac{1}{v l-T\left(p_{\perp}\right)+i \epsilon}=\frac{1}{v l+i \epsilon} \sum\left[\frac{T\left(p_{\perp}\right)}{v l+i \epsilon}\right]^{n}
$$

converges only for $T\left(p_{\perp}\right) / v l<1$. In the instance of $\pi N \rightarrow \pi N$ scattering and in the limit $T\left(p_{\perp}\right) \ll v l$ this series converges rather well. However for a production process $N N \rightarrow N N \pi$, the virtual nucleon in the graph $2 \mathrm{~b}$ has a residual momentum $l^{\prime}=\left(-m / 2, \mathbf{l}^{\prime}\right) ; \quad$ with $\quad \mathbf{l}^{\prime} \cdot \mathbf{l}^{\prime}=M m$, so that $T\left(p_{\perp}\right) / v l^{\prime} \approx-1$. Thus the corrections corresponding to each of the terms in Eqn. 5 are all of the same magnitude, i.e.,the power series of the nucleon propagator in the graph $2 \mathrm{~b}$ is on the border of its convergence circle and can not be approximated by any finite sum. Consequently, the HFF can not possibly predict the u channel impulse term correctly.

This same conclusion holds for one loop diagrams also. As an example the contribution from the loop diagram of Fig. 2c to the off mass shell $\pi N$ elastic scattering amplitude has the form,

$$
\begin{aligned}
& T_{\text {loop }}=\frac{2 g_{A}^{2}}{3 F^{4}}\left[3 Q^{2}-q^{2}-k^{2}+\frac{m^{2}}{2}\right] N^{\dagger}\left(p_{2}\right) S^{\nu} S^{\mu} N\left(p_{1}\right) \\
& \int D q^{\prime} q_{\mu}^{\prime}\left(q_{\nu}^{\prime}+Q_{\nu}\right) \tilde{S}_{N}\left(p_{2}-q^{\prime}\right)\left[\left(q^{\prime 2}-m^{2}\right)\left[\left(q^{\prime}+Q\right)^{2}-m^{2}\right]\right]^{-1},
\end{aligned}
$$

where $\tilde{S}_{N}(l)$ stands for the positive energy part of the nucleon propagator, i.e.,

$$
\tilde{S}_{N}(l)=i \frac{1}{v l+i \epsilon} \sum_{n}\left(\frac{(v l)^{2}-l^{2}}{2 M(v l+i \epsilon)}\right)^{n}+\ldots
$$

In our notations

$$
D q^{\prime}=\frac{\lambda^{4-d}}{(2 \pi)^{d}} d q^{\prime}
$$

with $\lambda$ being the scale of dimensional regularization, $k$ and $q$ are four momenta of the incoming and outgoing pions (see Fig. 2c), $Q \equiv\left(Q^{0}, \vec{Q}\right)$ is the transferred momentum $Q^{2}=\left(p_{2}-p_{4}\right)^{2}=(v Q)^{2}-\vec{Q}^{2}, S^{\mu}$ is the nucleon spin-operator and $\mathrm{F}$ and $g_{A}$ are the pion decay and axial-vector coupling constants, respectively.

To simplify calculations, we take $\vec{p}_{4}=0, \quad Q^{2}<0, \quad v Q=0$, values corresponding to the reaction $N N \rightarrow N N \pi$ at threshold. After straightforward though long and tedious calculations, the $n$-th correction term of the amplitude, Eqn. 6 is,

$$
\begin{aligned}
& T_{\text {loop }}^{(n)}=\frac{g_{A}^{2} m}{6 F^{4}}\left[3 Q^{2}-q^{2}-k^{2}+\frac{m^{2}}{2}\right] N^{\dagger}\left(p_{2}\right) N\left(p_{1}\right) \\
& \frac{i}{16 \pi^{2}}\left[A_{1}\left(Q^{2} / m^{2}\right)+\frac{-Q^{2}}{m^{2}}\left(A_{2}\left(Q^{2} / m^{2}\right)+A_{3}\left(Q^{2} / m^{2}\right)\right)\right],
\end{aligned}
$$

where,

$$
\begin{aligned}
& A_{1}(X)=\left(\frac{m}{M}\right)^{n}\left(\frac{m^{2}}{4 \pi \lambda^{2}}\right)^{-\epsilon} \frac{\Gamma(2-\epsilon) \Gamma\left(\frac{n+1}{2}\right)}{\Gamma\left(\frac{3}{2}-\epsilon\right)} \\
& \sum_{k, r} \frac{\Gamma\left(\frac{5}{2}-\epsilon+n-k-r\right) \Gamma\left(-\frac{n+1}{2}+\epsilon+k+r\right)}{\Gamma(n-2 k-r+1) \Gamma(k+3-\epsilon) \Gamma(2 k+1) \Gamma(r+1)} \\
& X^{k+r} F\left(2(k+r), \frac{n+1}{2}-\epsilon-k-r, X\right), \\
& A_{2}(X)=-\left(\frac{m}{M}\right)^{n}\left(\frac{m^{2}}{4 \pi \lambda^{2}}\right)^{-\epsilon} \frac{\Gamma(2-\epsilon) \Gamma\left(\frac{n+1}{2}\right)}{\Gamma\left(\frac{3}{2}-\epsilon\right)} \\
& \sum_{k, r} \frac{\Gamma\left(\frac{3}{2}-\epsilon+n-k-r\right) \Gamma\left(-\frac{n-1}{2}+\epsilon+k+r\right)}{\left(\frac{n+1}{2}-\epsilon-k-r\right) \Gamma(n-2 k-r+1) \Gamma(k+3-\epsilon) \Gamma(2 k+1) \Gamma(r+1)} \\
& X^{k+r} \frac{\partial}{\partial X} F\left(2(k+r), \frac{n+1}{2}-\epsilon-k-r, X\right),
\end{aligned}
$$




$$
\begin{aligned}
& A_{3}(X)=\left(\frac{m}{M}\right)^{n}\left(\frac{m^{2}}{4 \pi \lambda^{2}}\right)^{-\epsilon} \frac{\Gamma(2-\epsilon) \Gamma\left(\frac{n+1}{2}\right)}{\Gamma\left(\frac{3}{2}-\epsilon\right)} \\
& \sum_{k, r} \frac{\Gamma\left(\frac{3}{2}-\epsilon+n-k-r\right) \Gamma\left(-\frac{n-1}{2}+\epsilon+k+r\right)(2 k+1)}{\Gamma(n-2 k-r+1) \Gamma(k+3-\epsilon) \Gamma(2 k+1) \Gamma(r+1)} \\
& X^{k+r} F\left(2(k+r), \frac{n-1}{2}-\epsilon-k-r, X\right),
\end{aligned}
$$

with $\epsilon=(4-d) / 2$ and

$$
F(a, b, X)=\int_{0}^{1} d z z^{a}(1-X z(1-z))^{b} .
$$

The $\Gamma$-functions in the rhs of Eqns. 10, 11, 12 show that for even $n=2 n^{\prime}$ the functions $A_{i}$ are finite for $\epsilon \rightarrow 0$ and that for odd $n=2 n^{\prime}+1$ they have simple poles for such $k$ and $r$ which satisfy $-\frac{n \pm 1}{2}+\epsilon+k+r \leq 0$. As usual divergent terms must be renormalized introducing corresponding counterterms. We do not enter here into details of the renormalization procedure, but note that the finite terms, divergent terms as well as counterterms undergo this same small momentum expansion problem.

In the limit of small transferred momentum $-Q^{2} \leq m^{2}$, i.e., $-\left(Q^{2} / m^{2}\right) \leq 1$ and $F\left(a, b,-Q^{2} / m^{2}\right) \sim 1$, the $n$-th correction term becomes of the order $\sim(m / M)^{n}$ in agreement with the organizing principle of HFF $\chi$ PT.

The situation changes drastically at sufficiently large momentum transfer, say at threshold of the production process where $-Q^{2} \geq M m$. From Eqns.10-12 it is easy to see that the terms with $r=n, k=0$ can be dangerous as they are of order $\left(-Q^{2} / m M\right)^{n} \geq 1$. Provided the corresponding functions $F\left(a, b, Q^{2} / m^{2}\right)$ do not restore the small factor $\left(-m^{2} / Q^{2}\right)^{n}$, i.e. if, for example $F\left(2 n,-(n-1) / 2-\epsilon, Q^{2} / m^{2}\right) \nsim\left(-Q^{2} / m^{2}\right)^{-n}$, such terms would violate the HFF $\chi \mathrm{PT}$ organizing principle.

Let us now estimate the functions $F\left(2 n,-\frac{n-1}{2}-\epsilon, Q^{2} / m^{2}\right)$. For $n=1$ and $n=2$ these terms are of order of magnitude $\sim \Gamma(\epsilon)\left(Q^{2} / M m\right)$ and $\sim \Gamma(\epsilon)\left(Q^{2} / M m\right)^{3 / 2}(m / M)^{1 / 2}$, respectively. For $n \geq 3$ the dominant contribution to all these dangerous terms is finite and we may set $\epsilon=0$ in order to estimate the functions $F\left(2 n,-\frac{n-1}{2}, X\right)$ at large $X$. For even $n=2 n^{\prime}+2$ one obtains,

$$
\begin{aligned}
& F\left(4 n^{\prime}+4,-1 / 2-n^{\prime}, X\right)=\int_{0}^{1} d z \frac{z^{4 n^{\prime}+4}}{(1-X z(1-z))^{-1 / 2-n^{\prime}}}= \\
& 2^{-4 n^{\prime}+3}\left(-\frac{X}{4}\right)^{-1 / 2-n^{\prime}} \sum_{p=0}^{2 n^{\prime}+2} \sum_{r=0}^{p} \frac{\left(4 n^{\prime}+4\right) !}{\left(4 n^{\prime}+4-2 p\right) !(2 p) !} \\
& \frac{p !}{(p-r) ! r !} \sigma^{2 r} \int_{0}^{1} d t\left(\sigma^{2}-t^{2}\right)^{-1 / 2-n^{\prime}+p-r},
\end{aligned}
$$

where $\sigma^{2}=1-4 / X$. In the case where the parameter $X>>1$, the last integral may be expanded in powers of $1 / X$ with a dominant term being equal to $(1 / 2)(-X / 4)^{n^{\prime}+r-p-1 / 2}$. Hence the sum in the rhs of Eqn.14 can be written in the same power form. The largest term occurs for $r=p$, i.e.,

$$
F\left(4 n^{\prime}+4,-1 / 2-n^{\prime}, X\right) \approx \frac{1}{8\left(2 n^{\prime}+1\right)}\left(-\frac{1}{X}\right)
$$

for all $n^{\prime}=1,2, \ldots$. Similarly, for odd $n=2 n^{\prime}+1, \quad n^{\prime} \geq 1$ we have

$$
\begin{aligned}
& F\left(4 n^{\prime}+2,-n^{\prime}, X\right)=\int_{0}^{1} d z \frac{z^{4 n^{\prime}+2}}{(1-X z(1-z))^{n^{\prime}}}= \\
& 2^{-4 n^{\prime}+3}\left(-\frac{X}{4}\right)^{-n^{\prime}} \sum_{p=0}^{2 n^{\prime}} \sum_{r=0}^{p} \frac{\left(4 n^{\prime}+2\right) !}{(4 n+2-2 p) !(2 p) !} \\
& \frac{p !}{(p-r) ! r !} \sigma^{2 r} \int_{0}^{1} d t\left(\sigma^{2}-t^{2}\right)^{-n^{\prime}+p-r} \\
& \approx \frac{1}{8\left(2 n^{\prime}-1\right)}\left(-\frac{1}{X}\right) .
\end{aligned}
$$


Then one may conclude that for $n \geq 3$ the main contributions to $A_{1}\left(Q^{2} / m^{2}\right)$ have the same order in $-Q^{2} / m^{2}$ and write,

$$
A_{1}\left(Q^{2} / m^{2}\right) \approx \mathcal{A}_{1}(n) \frac{m^{2}}{-Q^{2}}
$$

In this last expression, the dependence on the correction order is incorporated in the coefficient $\mathcal{A}_{1}(n)$. For even and odd $n$ values these are $\mathcal{A}_{1}(n)=(3 / 256) \Gamma((n-1) / 2) \Gamma(n / 2) \Gamma(n+1)$ and $\mathcal{A}_{1}(n)=(3 / 256) \Gamma((n-1) / 2) \Gamma((n+$ $1) / 2) /(n / 2-1) \Gamma(n+1)$, respectively. we have found that at least one term on the rhs of Eqn.10 is of the order $m^{2} /\left(-Q^{2}\right)$ for all $n \geq 3$. In a similar way one estimates for $n \geq 1$,

$$
\begin{aligned}
& A_{2}\left(Q^{2} / m^{2}\right) \approx \mathcal{A}_{2}(n)\left(\frac{m^{2}}{Q^{2}}\right)^{2}, \\
& A_{3}\left(Q^{2} / m^{2}\right) \approx \mathcal{A}_{3}(n)\left(\frac{m^{2}}{Q^{2}}\right),
\end{aligned}
$$

where $\mathcal{A}_{2}(n)$ and $\mathcal{A}_{3}(n)$ incorporate as above all dependence of $A_{2}$ and $A_{3}$ on $n$. By substituting Eqns. 17, 18 and 19 into Eqn. 90 one finds that the $n$-th correction term is of order $\left(Q^{2} / m M\right)^{n}$. At threshold of the pion production process, $Q^{2}=-m M$ and each correction term $T_{\text {Loop }}^{(n)}$ involves a contribution of the same low momentum power order independently of $n$. This completes our proof that for the one loop graph 2c at large momentum transfer $-Q^{2}>>m^{2}$ the one to one correspondence between small momentum expansion and HFF corrections is broken, thereby violating the fundamental organizing principle of HFF $\chi \mathrm{PT}$.

\section{POWER COUNTING CONSIDERATIONS}

The standard Weinberg's power counting [18], where presumably the momentum transferred is of the order $Q^{2} \approx m^{2}$ can not be applied directly to meson production. Cohen et al, [4] suggested a modified scheme tailored to the production process. This scheme includes the following rules :

(i) a $\pi N N$ vertex of zero chiral order $\mathrm{D}=0, V_{\pi N N}^{(0)}$, contributes a factor $Q / F$,

(ii) a pion propagator contributes a factor $\left(Q^{2}\right)$,

(iii) a nucleon propagator $(v Q)^{-1} \approx m^{-1}$,

(iv) a $\pi N N$ vertex of chiral order $\mathrm{D}=1, V_{\pi N N}^{(1)}$ contributes a factor $m^{3 / 2} / F M^{1 / 2}$

(v) a $2 \pi \mathrm{NN} \mathrm{D}=1$ vertex, $V_{\pi \pi N N}^{(1)}$, contributes a factor $k^{0} Q^{0} / F^{2} M$.

To account for loop contributions also, one needs three more rules [6]:

(vi) a loop integration contributes a factor $\left(Q^{2} / 4 \pi\right)^{2}$.

(vii) a four pion vertex of zero order, $V_{\pi \pi \pi \pi}^{(0)}$, contributes a factor $Q^{2} / F^{2} \sim m M / F^{2}$.

(viii) a $3 \pi \mathrm{N}$ zero order vertex, $V_{\pi \pi \pi N N}^{(0)}$, contributes a factor $Q / F^{3} \sim \sqrt{m M} / F^{3}$.

The last two of these factors originate from the terms $\pi^{2}\left(\partial_{\mu} \pi\right)^{2} / 6 F^{2}$ and $S^{\mu} \pi^{2} \partial_{\mu} \pi / 6 F^{3}$ in the lowest-order Lagrangian, respectively. (see for example Eqn. 2 of Ref. [6])

The rule (vi) deserves some comments. We follow the procedure of loop calculations suggested by Park et al. [1] and use the dimensional regularization scheme (DRS). Using the parameterization scheme of Park et al. [i] and shifting the momentum variable after Wick rotation the loop integrand assumes the form,

$$
K_{\{\mu\},\{\nu\},\{\lambda\}} f\left(k^{2}\right) k^{d-1} d k d \Omega_{D},
$$

where the numerator $K_{\{\mu\},\{\nu\},\{\lambda\}}=\prod k_{\mu} v_{\nu} Q_{\lambda}$ absorbs all tensor structure of the integrand. Since $K_{\{\mu\},\{\nu\},\{\lambda\}}$ is multiplied by a product of nucleon spin matrices $\prod S_{\nu}$ the only terms which do not contain the nucleon velocity, $v_{\nu}$, may contribute. The denominator of the loop integral is a power of $\left(k^{2}+Q^{2} f_{q}+m^{2} f_{m}\right)$ in which the coefficients $f_{q}$ and $f_{m}$ do not depend on $k^{2}, Q^{2}$ and $m^{2}$. Therefore, a simple rescalling, $k^{2}=Q^{2} z^{2}$, enables to express the loop integral as a power expansion in $m^{2} / Q^{2}$. Since in the DRS all divergences separate from the remaining integrals as the poles at $\epsilon=(4-d) / 2 \rightarrow 0$, it follows that the loop integration gives a factor $Q^{4} /(4 \pi)^{2}$. In addition, to estimate contributions corresponding to higher power of $Q$ it is sufficient to take all internal pion momenta of $N N \pi$ vertices equal to $Q$. The corresponding loop integrals are found to be finite. 
Using these power counting rules the $n$-th correction term $T_{\text {Loop }}^{(n)}$ may be estimated as follows: loop integration contributes a factor $Q^{4} /(4 \pi)^{2}$, the $\pi N N$ vertices a factor $Q^{2} / F^{2}, 4 \pi$-vertex a factor $Q^{2} / F^{2}$, the pion propagators give $Q^{-4}$ and $n$-th order correction of the nucleon propagator a factor $(1 / v Q)\left(Q^{2} / 2 v Q\right)^{n}$. For the one loop diagram 2c one obtains,

$$
T_{\text {Loop }}^{(n)} \sim \frac{Q^{4}}{F^{2}(4 \pi F)^{2} v Q}\left[\frac{Q^{2}}{M v Q}\right]^{n} \sim \frac{1}{F}
$$

which is of the same order of magnitude as the first (uncorrected) term $T_{\text {Loop }}^{(0)}$. Again from power counting rules we reach the conclusion that for large momentum transfer processes the basic organizing principle of $\mathrm{HFF} \chi \mathrm{PT}$ is badly violated.

We now turn to demonstrate that for each low chiral order diagram contributing to the $N N \rightarrow N N \pi$ amplitude there exist infinite sequence of loop diagrams of higher chiral order, which have the same low momentum power as the original diagram [11]. Consider for example the diagrams shown in Fig. 3. The simplest nonvanishing at threshold irreducible diagram is the impulse term, (graph 1a), corresponding to a chiral order $D=1$, of which the

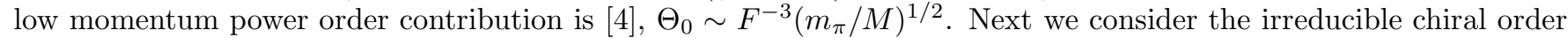
$D=3$ one loop diagram 3a which is obtained from diagram 1a by adding two $\pi N N \quad D=0$ vertices, two lowest order nucleon propagators and one meson propagator. From rule (i) above, a $\pi N N \quad D=0$ vertex is proportional to the meson three momentum, i.e.,

$$
V_{\pi N N}=\frac{g_{A}}{F} S Q \tau
$$

where $\mathrm{S}$ is the nucleon spin-operator. The two added nucleon vertices contribute a factor $Q^{2} F^{-2}$ and the two nucleon propagators give $(v Q)^{-2}$. Likewise, a meson propagator contributes a factor $Q^{-2}$ and the loop integral contributes a factor of $Q^{4} /(4 \pi)^{2}$. Altogether, diagram 3b has an additional factor $Q^{4}(4 \pi F v Q)^{-2}$ with respect to tree diagram 1a. The power factor of diagram $3 \mathrm{a}$ is therefore,

$$
\Theta_{3}=\Theta_{1} \frac{Q^{4}}{(4 \pi F)^{2}(v Q)^{2}} .
$$

With $4 \pi F \sim M, \quad v Q \sim m$, the diagram 3a is of the same order as the diagram 1a, i.e. $\Theta_{3}=\Theta_{1}$, though higher in chiral order. Similarly, by adding progressively, two zero order $\pi N N$ vertices, a pion propagator and two nucleon propagators, as mentioned above, one constructs the other higher order irreducible n-loop diagrams shown in Fig. 3.

By making use of the same power counting rules as above, the momentum power of the n-loop diagram would be,

$$
\Theta_{2 n+1}=\Theta_{1}\left(\frac{Q^{4}}{(4 \pi f)^{2}(v Q)^{2}}\right)^{n}=\Theta_{1} .
$$

We can thus construct an infinite sequence of n-loop diagrams, $n=1,2, \ldots$ of chiral order $2 n+1$ all having the same characteristic momentum power as the lowest chiral order impulse diagram 1a. Quite obviously, such a sequence of loops, does exist for any irreducible diagram that may contribute to the production process,and therefore we conclude that the small momentum power expansion scheme as a basic organizing principle of HFF $\chi \mathrm{PT}$ is badly violated in the instance of large momentum transfer.

\section{SUMMARY AND DISCUSSION}

We have considered tree and one loop diagram contributions to pion production in NN collisions using the nonrelativistic HFF. We have demonstrated that the positive energy part of the nucleon propagator, and in turn the corresponding positive energy contributions to tree level impulse $\mathrm{u}$ channel diagrams and one loop diagrams, are on the border of their convergence circles. Also, the basic principle of the HFF $\chi \mathrm{PT}$ of one-to-one correspondence between the loop and low momentum expansion is badly destroyed. The primary production amplitude becomes the sum over infinite sequences of diagrams. This excludes the possibility that a finite chiral order HFF based $\chi \mathrm{PT}$ calculations can explain meson production in NN collisions.

Acknowledgments This work was supported in part by the Israel Ministry Of Absorption. 
[1] T.S. Park, D.-P. Min and M.Rho, Phys. Rep. 233, 341 (1993).

[2] V.Bernard, N.Kaiser and Ulf-G.Meissner, Int.J.Mod.Phys.E4,193, (1995)

[3] B.-Y.Park et al., Phys. Rev. C53, 1519 (1996).

[4] T.D.Cohen et al., Phys. Rev. C53, 2661 (1996).

[5] T.Sato et al., Phys. Rev. C56, 1246 (1997).

[6] E.Gedalin, A.Moalem and L.Razdolskaya, nucl-th/9803029, Phys. Rev.C 60, 031001 (1999).

[7] V. Bernard, N. Kaiser and Ulf-G.Meissner, Eur.Phys.J.A4(1999) 259.

[8] E.Gedalin, A.Moalem and L.Razdolskaya, nucl-th/9812009.

[9] V.Dmitrašinović, K.Kubodera,F.Myhrer and T.Sato, nucl-th/9902048.

[10] C.A.da Rocha, G.A.Miller, U.van Kolck, nucl-th/9904031.

[11] E.Gedalin, A.Moalem and L.Razdolskaya, nucl-th/9906025.

[12] E.Hernandez and E.Oset, Phys.Lett.B 350,(1995) 158.

[13] C.Hanhart et al.,Phys.Lett.B 358, (1995) 21.

[14] E. Gedalin, A. Moalem and L. Razdolskaya, nucl-th/9611005, Nucl. Phys. A652(1999) 287.

[15] C.Hanhart et al.,. Phys.Lett. B 424,(1998) 8.

[16] K.Tamura et al., To be published and private communication with A. Moalem.

[17] V. De Alfaro, S. Fibini, G. Furlan, C. Rossetti, "Currents In Hadron Physics", North-Holland, Amsterdam 1973, p. 171.

[18] S.Weinberg, Physica, 96A,(1979) 327. 


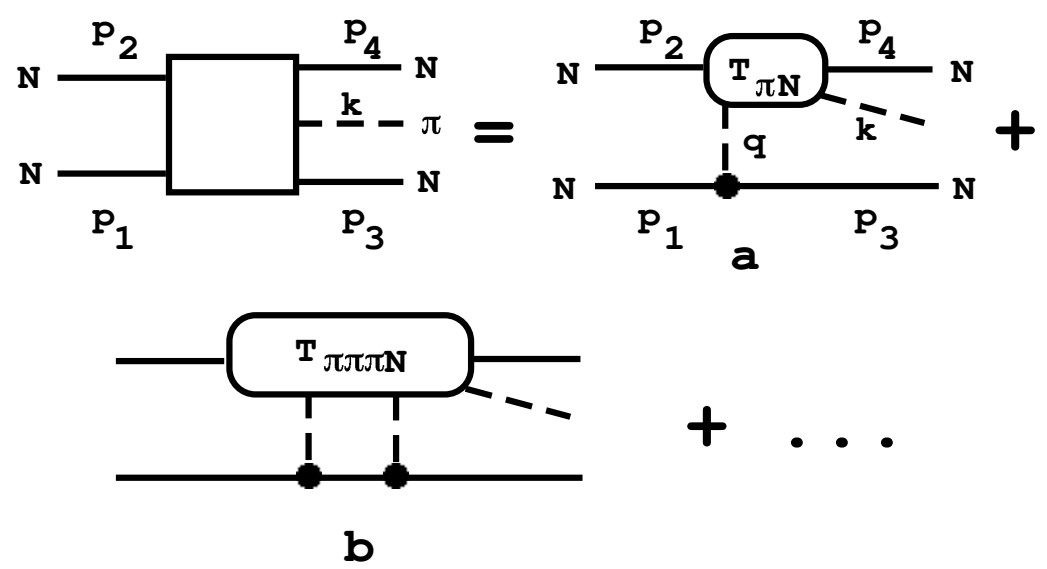

FIG. 1. One pion exchange ( diagram a) and two pion exchange (diagram b) contributing to the $N N \rightarrow N N \pi^{0}$ reaction. Nucleons are represented by solid lines and mesons by dashed lines. $T_{\pi N}$ denotes the off-shell pion-nucleon elastic scattering amplitude. $T_{\pi \pi \pi N}$ denotes the off-shell $\pi N \rightarrow \pi \pi N$ amplitude. 

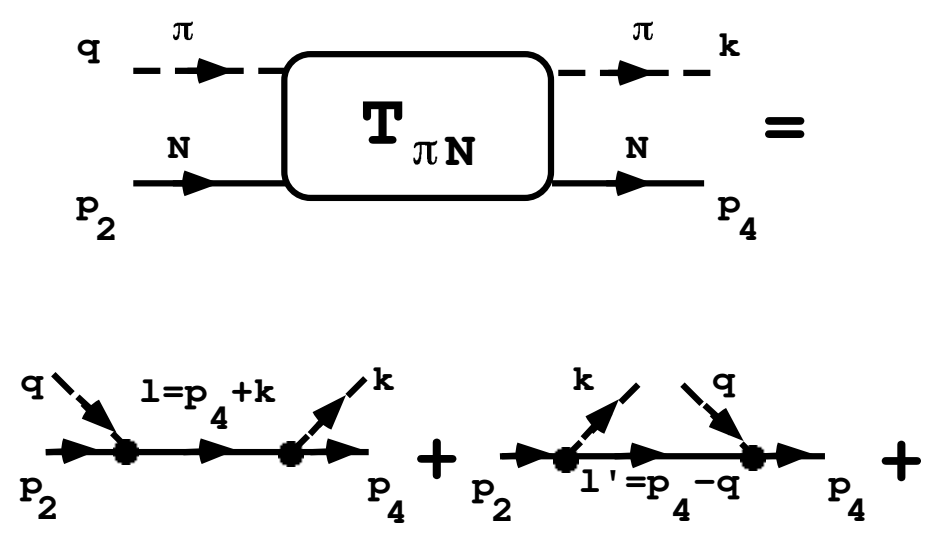

a b

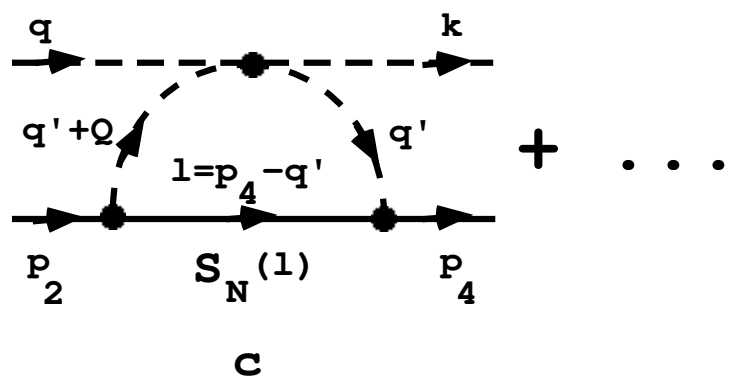

FIG. 2. Pole terms (diagrams (a) and (b)) and loop (diagram c) contributions to the off mass shell $\pi N \rightarrow \pi N$ scattering amplitude. 


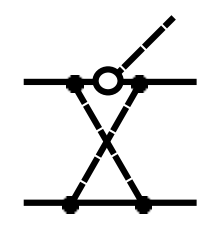

a

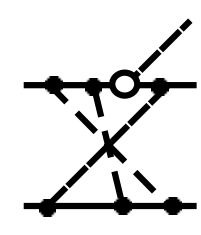

C

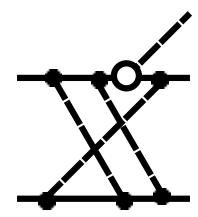

b

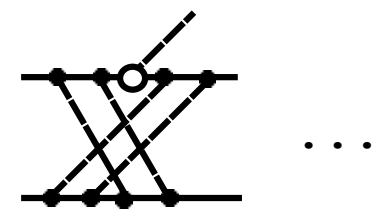

d

FIG. 3. An infinite sequence of diagrams contributing to the pion production process (see text). Black dots and open circle are zero and 1 chiral order $\pi N N$ vertices, respectively. Shown in the figure are irreducible one loop (diagram a), two-loop (diagrams b and c) and one of the three-loop (diagram d). The ellipsis denote all other loop diagrams of the sequence. 